\title{
Philosophiques
}

\section{Précis de Catching Capital - The Ethics of Tax Competition}

\section{Peter Dietsch}

Volume 43, numéro 1, printemps 2016

URI : https://id.erudit.org/iderudit/1036470ar

DOI : https://doi.org/10.7202/1036470ar

Aller au sommaire du numéro

Éditeur(s)

Société de philosophie du Québec

ISSN

0316-2923 (imprimé)

1492-1391 (numérique)

Découvrir la revue

Citer ce document

Dietsch, P. (2016). Précis de Catching Capital - The Ethics of Tax Competition. Philosophiques, 43(1), 115-118. https://doi.org/10.7202/1036470ar d'utilisation que vous pouvez consulter en ligne.

https://apropos.erudit.org/fr/usagers/politique-dutilisation/ 


\title{
Disputatio
}

\section{Précis de Catching Capital - The Ethics of Tax Competition'}

\author{
PETER DIETSCH \\ Université de Montréal
}

La mobilité du capital représente un enjeu majeur de la globalisation économique. Différents agents économiques exploitent cette mobilité, notamment, pour minimiser leur fardeau fiscal. Pensons aux particuliers qui se prévalent du secret bancaire ou de l'obscurité des « trusts » pour cacher leur richesse dans des paradis fiscaux. Une telle évasion est bien évidemment illégale. Des firmes transnationales (FTN), quant à elles, utilisent toute une gamme de techniques pour déplacer les profits de leurs activités économiques vers des juridictions ayant un faible fardeau fiscal. Contrairement à l'évasion des particuliers, les différents types d'évitement fiscal restent pour la plupart légaux. Par ailleurs, les FTN décident parfois de délocaliser l'activité économique elle-même vers un pays imposant moins fortement le capital.

Ce serait trop facile de blâmer seulement les individus et les FTN pour l'utilisation de telles pratiques. Ils répondent à une structure d'incitatifs économiques mise en place et tolérée par les États. Ces derniers utilisent en fait leur politique fiscale pour attirer le capital des particuliers et des FTN dans leur juridiction: un tel phénomène fait référence à ce qu'on appelle communément la concurrence fiscale entre les États. On peut définir celle-ci comme étant la détermination de la politique fiscale des gouvernements indépendants œuvrant dans un contexte non coopératif et stratégique.

Plusieurs raisons expliquent que cette concurrence fiscale pose problème d'un point de vue éthique. Elle nuit d'abord à la capacité des communautés politiques de taxer le capital de la manière qui correspond à leurs préférences politiques. En effet, en permettant aux propriétaires d'un capital de le déplacer dans une autre juridiction sans déménager, la concurrence fiscale mine le contrat social et la vie politico-économique telle que nous la connaissons. Un individu riche ou une FTN très lucrative peuvent désormais profiter des biens publics d'un pays sans contribuer à leur juste part de financement. C'est sans compter la tendance de la concurrence fiscale à exacerber les inégalités déjà énormes de revenu et de richesse dans nos sociétés, ainsi qu'à l'échelle globale.

1. Oxford University Press, 2015.

PHILOSOPHIQUES 43/1 — Printemps 2016, p. 115-118 
Dans mon livre Catching Capital - The Ethics of Tax Competition, je propose une évaluation en trois étapes de la concurrence fiscale d'un point de vue éthique. D'abord, je présente une analyse de la dynamique de la concurrence fiscale et de son impact sur les valeurs sociales pertinentes (chapitre I), pour ensuite développer des principes normatifs et des règles institutionnelles propices à contrer l'effet nocif de cette concurrence (chapitre 2), pour terminer avec une défense de la régulation proposée contre des objections basées sur l'efficience et la souveraineté (chapitres 3 et 4 ) ainsi qu'une analyse des questions de justice transitionnelle posées par le cadre régulateur proposé (chapitre 5). Mon livre innove par son analyse de la dynamique interne de la fiscalité internationale qui se fait à la fois d'un point de vue éthique et en prenant au sérieux l'interdisciplinarité de la question.

Dans cette introduction à ce symposium, je donnerai un bref résumé du livre chapitre par chapitre en soulignant au passage des points importants pour la compréhension du projet dans son ensemble.

$\mathrm{Au}$ début du premier chapitre, je précise que mon évaluation normative de la concurrence fiscale ne se basera que sur une seule valeur, à savoir l'autonomie fiscale - et non pas, notamment, sur une théorie de la justice distributive globale. Ce choix aura des répercussions importantes pour les conclusions de l'analyse ${ }^{2}$. L'autonomie fiscale fait référence à la capacité des communautés politiques de choisir la taille de leur État par rapport au PIB ainsi que le niveau de redistribution effectuée entre leurs citoyens.

L'argument du premier chapitre procède en deux étapes principales. D'abord, je distingue trois sortes de concurrence fiscale visant différents types de capital: I) le capital de portefeuille des particuliers (exemple: les paradis fiscaux de la Suisse ou des Îles Caïmans et leur culture du secret); 2) le profit sur papier des entreprises (exemple: les boîtes de brevets; l'abus du prix de transfert, des structures de dette entre des succursales de FTN, etc.); et 3) l'investissement direct à l'étranger (IDE) (exemple: le taux d'imposition de $\mathrm{I} 2.5 \%$ de l'Irlande sur les profits d'entreprise). Les deux premiers se distinguent par le fait que le particulier et la FTN en question ne se déplacent pas physiquement; c'est seulement leur capital qui est envoyé ailleurs. On peut dire qu'ils sont des «resquilleurs» (free-riders) sur le contrat social de leur pays de résidence ou d'activité économique, et que l'État attirant leur capital pratique du «braconnage» (poaching) sur le territoire fiscal d'un autre État. Le cas de l'IDE se distingue dans la mesure où il s'agit plutôt de "leurrer»(luring) des agents économiques à part entière dans une autre juridiction.

La deuxième partie du chapitre vise à montrer que la concurrence mine le principe d'autonomie fiscale. En réponse à la pression sur les taux d'imposition du capital, les pays développés ont déplacé le fardeau fiscal

2. Je commenterai davantage ces répercussions dans la réponse au commentaire de Louis-Philippe Hodgson. 
vers la consommation ou le travail, ce qui leur a permis de conserver leur niveau de revenu. Mais une telle manœuvre ne se fait pas sans coûts, le système fiscal devenant plus régressif en imposant alors un sacrifice sur l'idéal redistributif qui représente le deuxième aspect de l'autonomie fiscale. Les pays en développement se retrouvent dans une pire situation encore, en perdant des revenus en sus.

Le chapitre 2 est construit sur cette analyse et propose deux principes de justice fiscale globale (voir aussi Dietsch \& Rixen, 20I4). Premièrement, je défends un principe d'appartenance (membership principle), qui exige que toute personne, physique et morale, doive payer ses impôts dans la juridiction où elle est membre. Dans le cas des particuliers, on peut penser au lieu de résidence; en ce qui a trait aux FTN, à l'endroit dans lequel se déroule l'activité économique. Ce principe correspond à l'esprit des lois de fiscalité internationale appliquées à l'heure actuelle, mais l'existence de maintes échappatoires fait qu'elles sont souvent violées. Il faut souligner que cela implique aussi l'interdiction complète des deux formes de braconnage (la concurrence fiscale pour le capital de portefeuille ainsi que pour les profits sur papier des FTN).

Si l'on imagine un monde sans braconnage, on se rend compte rapidement que l'incitatif pour les agents économiques de réellement changer de résidence ou de lieu de production augmentera de façon drastique. Il existe une relation de substitution entre le braconnage et les pratiques de leurre, ce qui vient souligner l'importance de la question de la justice de ces pratiques. C'est dans cette optique que nous proposons la contrainte sur la politique fiscale (fiscal policy constraint) comme deuxième principe de justice fiscale globale. Cette contrainte spécifie que toute politique fiscale à la fois motivée par des raisons stratégiques (au lieu de refléter les préférences politiques des citoyens) et réduisant l'autonomie fiscale agrégée des autres pays devrait être interdite. Ce dernier principe a l'effet de limiter les pratiques de leurre, sans toutefois les éliminer complètement ${ }^{3}$. Pour veiller au respect des deux principes décrits ci-dessus, je propose la création d'une Organisation mondiale de la fiscalité.

Le chapitre 3 répond à l'objection de la présumée inefficacité de la régulation proposée ci-dessus. Dans un premier temps, j’y présente une analyse critique des modèles économiques de la concurrence fiscale évaluant le phénomène selon la perspective de l'efficience de Pareto. Je montre que la question centrale de ces modèles, à savoir si la concurrence fiscale est Pareto optimale 4 , s'avère d'une pertinence pratique limitée, car leurs conclusions ne tiennent pas la route lorsqu'il s'agit d'appliquer les circonstances idéalisées des modèles au monde réel. La question pertinente est la suivante: la régu-

3. Voir mes réponses aux commentaires pour plus de détails.

4. Ce terme désigne une situation dans laquelle il n'est pas possible d'améliorer le bienêtre d'une personne donnée sans diminuer celui d'une autre. 
lation de la concurrence fiscale représente-t-elle une amélioration (ou une détérioration) de Pareto ? ${ }^{5}$ Étant donné qu'une telle régulation impliquerait nécessairement des gagnants et des perdants, on ne parlera jamais d'amélioration selon une telle définition. L'efficience dans le sens de Pareto ne peut donc pas être invoquée comme une objection à la réforme proposée.

Par ailleurs, le chapitre 3 montre également que la concurrence fiscale risque de miner l'efficience entendue dans un second sens, selon la théorie de la fiscalité optimale, en tant que balance des valeurs de la croissance économique et de l'idéal redistributif de la société. En montrant que la concurrence fiscale introduit un biais dans ce calcul, je fournis un argument supplémentaire pour sa régulation. Cette dernière ne se veut donc pas seulement une exigence de la justice, mais également un élément clé d'une politique économique raisonnable.

Dans le chapitre 4, sur lequel je serai très bref ici, je discute l'objection selon laquelle une régulation de la concurrence fiscale représente une entrave à la souveraineté fiscale des États. À mon avis, cette objection repose sur une compréhension dépassée de ce concept. Dans un contexte d'interdépendance économique, il ne fait effectivement plus de sens d'avancer une conception de la souveraineté axée sur le principe de non-interférence. Une fois reconnu le fait qu'elle n'implique pas seulement des droits, mais aussi des obligations envers d'autres États, une régulation de la concurrence fiscale émerge en tant qu'exigence de la souveraineté plutôt qu'en tant qu'obstacle à celle-ci.

Les questions éthiques sur la concurrence fiscale ne se limitant pas à l'identification d'une régulation juste ou optimale, des interrogations quant à l'antériorité et la postériorité d'une telle réforme entrent aussi en jeu dans le cadre de mon ouvrage. Le chapitre 5 est consacré à trois questions de ce type, que je regroupe sous le thème de la justice transitionnelle, et qui complémentent et nuancent les propos des quatre premiers chapitres. Premièrement, sous le statu quo, les gagnants de la concurrence fiscale ont-ils des obligations de compensation pour les perdants? Deuxièmement, avant qu'une régulation ne soit mise en place, est-il juste de contester aux pays en développement le droit de pratiquer la concurrence fiscale pour financer leur développement? Et troisièmement, en supposant que les réformes proposées dans mon livre soient adoptées, y a-t-il des conditions dans lesquelles des anciens paradis fiscaux auraient des revendications légitimes de compensation pour la perte de revenus d'emplois ou d'autres avantages?

5. Une redistribution de ressources qui améliore (réduit) le bien-être d'une personne donnée sans diminuer le bien-être d'une autre. 